\title{
More comprehensively measuring quality of life in life-threatening illness: the McGill Quality of Life Questionnaire - Expanded
}

\author{
S. Robin Cohen ${ }^{1,2^{*}} \mathbb{D}$, Lara B. Russell ${ }^{3}$, Anne Leis ${ }^{4}$, Javad Shahidi ${ }^{5}$, Pat Porterfield ${ }^{6}$, David R. Kuhl ${ }^{7}$, \\ Anne M. Gadermann ${ }^{3,8}$ and Richard Sawatzky ${ }^{3,9}$
}

\begin{abstract}
Background: Domains other than those commonly measured (physical, psychological, social, and sometimes existential/spiritual) are important to the quality of life of people with life-threatening illness. The McGill Quality of Life Questionnaire (MQOL) - Revised measures the four common domains. The aim of this study was to create a psychometrically sound instrument, MQOL - Expanded, to comprehensively measure quality of life by adding to MQOL-Revised the domains of cognition, healthcare, environment, (feeling like a) burden, and possibly, finance.

Methods: Confirmatory factor analyses were conducted on three datasets to ascertain whether seven new items belonged with existing MQOL-Revised domains, whether good model fit was obtained with their addition as five separate domains to MQOL-Revised, and whether a second-order factor representing overall quality of life was present. People with life-threatening illnesses (mainly cancer) or aged $>80$ were recruited from 15 healthcare sites in seven Canadian provinces. Settings included: palliative home care and inpatient units; acute care units; oncology outpatient clinics.

Results: Good model fit was obtained when adding each of the five domains separately to MQOL-Revised and for the nine correlated domains. Fit was acceptable for a second-order factor model. The financial domain was removed because of low importance. The resulting MQOL-Expanded is a 21-item instrument with eight domains (fit of eight correlated domains: Comparative Fit Index =.96; Root Mean Square Error of Approximation $=.033$ ).

Conclusions: MQOL-Expanded builds on MQOL-Revised to more comprehensively measure the quality of life of people with life-threatening illness. Our analyses provide validity evidence for the MQOL-Expanded domain and summary scores; the need for further validation research is discussed. Use of MQOL-Expanded will enable a more holistic understanding of the quality of life of people with a life-threatening illness and the impact of treatments and interventions upon it. It will allow for a better understanding of less commonly assessed but important life domains (cognition, healthcare, environment, feeling like a burden) and their relationship to the more commonly assessed domains (physical, psychological, social, existential/spiritual).
\end{abstract}

Keywords: Quality of life, Measurement, Psychometrics, End of life, Chronic disease, Palliative care, Burden of illness, Chronic illness

\footnotetext{
* Correspondence: robin.cohen@mcgill.ca

${ }^{1}$ Departments of Oncology and Medicine, McGill University, Montréal,

Canada

${ }^{2}$ Lady Davis Research Institute of the Jewish General Hospital, Palliative Care

Research, room E8.06, 3755 Côte Ste. Catherine Road, Montréal, Québec H3T

1E2, Canada

Full list of author information is available at the end of the article
}

(c) The Author(s). 2019 Open Access This article is distributed under the terms of the Creative Commons Attribution 4.0 International License (http://creativecommons.org/licenses/by/4.0/), which permits unrestricted use, distribution, and reproduction in any medium, provided you give appropriate credit to the original author(s) and the source, provide a link to the Creative Commons license, and indicate if changes were made. The Creative Commons Public Domain Dedication waiver (http://creativecommons.org/publicdomain/zero/1.0/) applies to the data made available in this article, unless otherwise stated. 


\section{Background}

A crucial starting point for the validity of any instrument measuring quality of life (QOL) is that it assesses the domains important to the population whose QOL it is designed to measure. Creating QOL instruments requires a balance between comprehensive coverage of what is important to the QOL of the population and feasibility of completion, since there are many potentially relevant life domains, and each has many aspects. Feasibility depends in part on where the respondent is in the disease trajectory. QOL instruments that might be used in the last few months of life necessarily need to be brief. When QOL is being measured alongside other variables, it may be necessary to measure only the domains most strongly related to QOL, especially when QOL is not the primary construct of interest. However, when QOL is the primary outcome, or it is feasible to have respondents complete a longer but still brief QOL questionnaire along with collection of other necessary data, it is important that the QOL measure be as comprehensive as possible. Domains widely accepted as essential for measuring the QOL of people who are ill include the physical, psychological/mental, and social - corresponding to the definition of health in the World Health Organization's constitution [1]. For people with a life-limiting illness, the existential or spiritual domain is increasingly considered essential as well $[2,3]$.

The McGill Quality of Life Questionnaire (MQOL), first published in 1996, was developed to measure the QOL (subjective well-being) of people with a lifethreatening illness throughout the illness trajectory. It assesses the four essential domains for this population: physical, psychological, support, and existential or spiritual (plus one global QOL item) [4, 5]. It has been widely used, in part because of its perceived high content validity and acceptability for people at the end of life [6, 7]. MQOL does not include items on the intensity of a variety of physical symptoms (for which a symptom measure can be used) but rather focuses on the impact of physical symptoms on QOL. Initial selection of MQOL's content was based on a review of pre-existing QOL instruments for people with cancer, the QOL literature at the time, clinical expertise, and understanding gained through comments patients made to S.R.C. in a study using other QOL instruments. Since QOL is subjective, content is ideally informed by the people whose QOL is to be measured. To improve MQOL, this step was undertaken by Cohen and Leis [8] in a qualitative study to learn from people at the end of life what is important to their QOL. That study revealed that MQOL is missing some domains important for a comprehensive QOL assessment. In addition to the original MQOL domains (physical, psychological, social, and existential), the person's environment (e.g. privacy; quiet; access to nature; care setting), cognitive functioning, the quality of healthcare, and a sense of being a burden are important to QOL at the end of life. Therefore, a program of research was undertaken to develop a more comprehensive instrument, MQOL-Expanded (MQOL-E), that includes all eight domains.

Several other published studies provide evidence as to which life domains are relevant to the QOL of people with a life-limiting illness (see McCaffrey et al. [3] for some examples). The findings across studies are not completely consistent: different domains are described in different papers, and what appear to be similar concepts are included in domains of different names in different articles. Therefore, in developing MQOL-E, we needed to make decisions about what concepts should be included. There were two primary considerations. 1) To include all domains found to be important in the Cohen and Leis study [8], since that study was the stimulus for the development of MQOL-E. 2) To keep MQOL-E brief enough to be feasible to complete by people who are in the last weeks of life. To keep it brief, we needed to select general content to reflect each domain, rather than include all specific aspects of each domain. The Cohen and Leis study is a good basis for content development because it is one of only two studies to report all eight domains found in a recent systematic review of important aspects of QOL as reported by palliative care patients. The eight domains described in that review are: physical; personal autonomy; emotional; social; spiritual (environment is included here); cognitive; healthcare; and preparatory (for death or to aid family afterward). ${ }^{1}$ [3] Some participants in the Cohen and Leis study mentioned that worrying about finances was stressful, but the data did not suggest elevating this to domain status. Since this theme focused on worry and stress, it was considered by the investigators to be captured in the anxiety item in MQOL. However, because financial concerns have been increasingly identified as a contributor to the QOL of people receiving palliative care [9-12], an item was developed and tested in one of the three datasets informing this paper to assess the extent to which it contributes to the concept of overall QOL [13]. There have been reports describing other issues that might be included in a QOL instrument for people with lifethreatening illness $[9,11,14-18]$, but other than the financial domain, none have as much support as the eight domains we selected to be included in MQOL-E.

\footnotetext{
${ }^{1}$ For simplicity, we have subsumed different authors' specific variations in domain name and content within a single broad domain where there is significant conceptual overlap. For example, despite differences between the existential and spiritual concepts, we have considered them to be one domain. Similarly, we combined the concepts of support, relationships, and social functioning and refer to them as the social domain.
} 
The studies to create MQOL-E also provided an opportunity to improve MQOL by addressing issues that became apparent with its use over time. If possible, we also wanted to reduce the number of items measuring the original four domains, to keep completion feasible despite the domains to be added for MQOL-E. We created a revised questionnaire, MQOL-Revised (MQOL-R) [19] onto which MQOL-E's new content is added, thus allowing comparison of the original four domains across future studies using either MQOL-R or MQOL-E.

This paper reports on the creation of MQOL-E, an instrument to more comprehensively measure the domains relevant to the QOL of people with a life-threatening illness. The aim was to add new subscales for the environment, cognition, healthcare, and a feeling of being a burden to MQOL-R's subscales (Physical, Psychological, Existential, Social), and to test the relevance of the financial domain to the concept of overall QOL.

\section{Methods}

\section{Item development for the new domains}

$\mathrm{SRC}, \mathrm{AL}$, and the late Terry Bunston, $\mathrm{PhD}$, with feedback from a larger research team, developed initial items to capture three new domains (Environment; Cognition; Healthcare) and the construct of (feeling like a) Burden. These were combined with select MQOL items to create a 32-item questionnaire. Like MQOL and MQOL-R, each MQOL-E item has a 2-day timeframe. Sixty palliative care patients from inpatient and home care settings in three Canadian cities and 10 palliative care clinicians then rated each item for importance to patient QOL $(0-$ 10 scale) and indicated items they found unclear (criterion of $\leq 10 \%$ for retention), redundant, or upsetting. For example, an earlier item stem, "I feel like a burden", was upsetting and unclear ("burden to whom?") to some participants, but they deemed the content important. It was revised to "I felt badly about how my situation affected the people I care about". Rejected items were replaced. The new items were tested with another 63 patients, who completed the revised 26-item questionnaire to evaluate item distribution and again assess clarity and redundancy. New revisions resulted in a 20 -item questionnaire. A validation study with this new questionnaire found the factor structure to be unsatisfactory since items representing different domains did not load as predicted ( $N=304$; unpublished). Various new changes and 11 experimental items, including one for the new financial domain, were explored in 23-27 item questionnaires in the three studies that provided datasets for this report. Since other instruments were completed at the same time in each of these studies $[13,20]$, to respect the physical condition of the participants, not all potential replacement items could be tested at once. To create the domain of quality of healthcare, Study G (the chronologically earliest dataset used in this report) used the two items from the immediately preceding 20 -item version and also tested three new items, since we were hoping to create items with improved distributions. The new items, which did not have substantially improved distributions, were not included in subsequent studies. Only the two older items, which had been reviewed by patients during the development of the 20-item version and for which more data were available (since they were used in studies $\mathrm{E}$ and $\mathrm{F}$ ), were included in the present analyses.

Items were initially developed in English, then translated into French by a professional translator. These two versions were compared by bilingual patients and clinicians; slight discrepancies between the two versions were reconciled. Sometimes this required a different word in French, other times the French translation suggested that the English wording needed clarifying, which was done. These changes did not affect the initial intent of the item. The resulting French version was successfully professionally back-translated into English with no further changes required. Both language versions were used in collecting datasets E and G, where participants chose the language version they preferred. Study F used only the English version since it was part of a larger study conducted in English in a city where English is the language of daily interaction.

\section{Items used in tests of construct validity}

To develop the final version of MQOL-E, we tested the seven new items representing the new domains and burden construct described in the Introduction and shown in Table 1 (the original 11 minus the four for quality of healthcare that were removed as explained above). As with MQOL-R, all items are measured on an 11-point scale from 0 to 10 , anchored as indicated.

\section{Datasets, participants and consent}

The datasets used in the analyses reported here (E-G) were collected from people with life-threatening illnesses at all stages of the disease trajectory or aged $>80$ years. They were recruited from 15 Canadian healthcare sites in seven provinces. Study E $(N=219)$ was designed to test the construct validity of a 24-item pre-cursor instrument to MQOL-E [13]. The primary purpose of Study F $(N=368)$ was to develop a self-report instrument to measure satisfaction with healthcare. A 23-item precursor instrument to MQOL-E was used in that study to assess the construct validity of the satisfaction with care instrument [20]. Study G $(N=216)$ used a 27 -item precursor to MQOL-E in a longitudinal study of the QOL of palliative care patients and was also intended to be used to assess construct validity (unpublished). Table 2 provides demographic information about the participants in each study. Datasets E and G include only 
Table 1 Domains and corresponding items tested for inclusion in MQOL-E

\begin{tabular}{lll}
\hline Domain & Item stem [time frame of past 2 days $(48 \mathrm{~h})$ not shown] & Item end anchors \\
\hline Healthcare & Getting the information I needed from the health care team was: & difficult/very easy \\
& The quality of health care I received was: & unsatisfactory/extremely good \\
Cognitive functioning & I was able to think clearly: & not often/always \\
& My memory worked: & noorly/very well \\
(Feeling like a) Burden & I felt badly about how my situation affected the people I care about: & not at all/completely \\
Environment & My physical surroundings met my needs & not at all/completely \\
Financial & My financial situation has been stressful &
\end{tabular}

people with cancer, while $\mathrm{F}$ includes people with various end-stage diseases and/or advanced age. Participants were recruited from a range of settings: palliative home care and inpatient units, acute care units, and oncology outpatient clinics. In studies $\mathrm{F}$ and $\mathrm{G}$, the mean age was over 65; similar numbers of women and men participated. Study E recruited from oncology clinics, where the participants were somewhat younger (mean 58 years) and $64 \%$ were women. More detailed information about the three study samples is provided in Table 2 .

In studies $\mathrm{F}$ and $\mathrm{G}$, written informed consent was provided; participants had the choice of completing the questionnaire on their own or as a structured interview. In study E, with permission of the Research Ethics Committee, potential participants were provided with an information sheet and questionnaire to be completed and returned anonymously; consent was implied if the questionnaire was completed. All of the studies for item development and the current analysis were approved by the research ethics boards of all of the institutions from which patients were recruited.

\section{Analysis}

Candidate items and domains were tested as additions to MQOL-R in four steps by conducting Confirmatory Factor Analyses (CFAs) using maximum likelihood robust estimation. The procedures, criteria for determining model fit, and goal of each step are described in Table 3. Analyses were conducted using the Mplus software [21]. Model fit was assessed via the Comparative Fit Index (CFI; >.95 indicates good fit) and the Root Mean Square Error of Approximation (RMSEA; <.06 indicates good fit) using criteria from $\mathrm{Hu}$ and Bentler [22]. The chi-square test was not used as it is sensitive to sample size [23]. To ensure that the items intended to represent new domains represented a construct different from existing MQOL-R domains, we first tested the factor loading of each item on existing domains. A standardized factor loading $\geq .6$ was considered to form part of an existing domain. This is higher than the usual minimum criterion of $\geq .4$ [24] because these items were designed to measure different aspects of QOL than the MQOL-R domains, therefore, adding them to an existing domain instead would require

Table 2 Demographics for each sample used in the development of MQOL-E

\begin{tabular}{|c|c|c|c|}
\hline \multirow[t]{2}{*}{ Variables } & \multicolumn{3}{|l|}{ Study ${ }^{a}$} \\
\hline & $E$ & $F^{c}$ & $\mathrm{G}^{\mathrm{d}}$ \\
\hline N & 219 & 368 & 216 \\
\hline Gender (\% female) & 64 & 48 & 51 \\
\hline Age (mean; standard deviation) & $58(15.4)$ & $77(9.9)$ & $66(12.5)$ \\
\hline \multicolumn{4}{|l|}{ Highest level of education (\%) } \\
\hline Did not complete high school & 10 & 46 & 32 \\
\hline High school completed but no post-secondary & 34 & 22 & 20 \\
\hline Some post-secondary & 56 & 30 & 42 \\
\hline Disease & Cancer & $\begin{array}{l}\text { Various end-stage diseases } \\
\text { (COPD; CHF; cirrhosis; cancer) } \\
\text { or }>80 \text { years old }\end{array}$ & Cancer \\
\hline Settings (\%) & Outpatient clinics (100) & $\begin{array}{l}\text { Acute care units (71) } \\
\text { Home care }(22) \\
\text { Palliative care units (7) }\end{array}$ & $\begin{array}{l}\text { Palliative care units }(71) \\
\text { Palliative home care (27) } \\
\text { Other (1) } \\
\text { Missing (1) }\end{array}$ \\
\hline
\end{tabular}

${ }^{a}$ Since these studies were also used in development of MQOL-R, the same naming convention was used [19].

${ }^{b}$ For Study E, participants reported the highest level of education attended. For other datasets, participants reported the highest level of education completed. ${ }^{c}$ Timeframe of 1 month rather than 2 days. ${ }^{d}$ Longitudinal study. The first questionnaire completed by each participant was selected to provide the largest $\mathrm{n}$ (i.e. before dropout) 
Table 3 Analysis methods and results

\begin{tabular}{|c|c|c|c|}
\hline Step & Goal of analysis & $\begin{array}{l}\text { Procedure and criteria for model and } \\
\text { item fit }\end{array}$ & Results \\
\hline 1 & $\begin{array}{l}\text { To ensure that new items, which were } \\
\text { conceived to represent new domains, } \\
\text { did not reflect existing MQOL-R } \\
\text { domains }\end{array}$ & $\begin{array}{l}\text { Each of } 7 \text { candidate items (see } \\
\text { Table 2) was added one at a time to } \\
\text { the MQOL-R model and allowed to } \\
\text { cross-load on all existing domains of } \\
\text { the MQOL-R } \\
\text { Criteria to conclude the item belongs to } \\
\text { an existing MQOL-R domain } \\
\text { - Comparative Fit Index (CFI) }>0.95 \\
\text { - Root Mean Square Error of } \\
\text { Approximation (RMSEA) }<0.06 \\
\text { - Standardized factor loadings } \geq 0.6 \text { on } \\
\text { existing domains. Loadings between } \\
4 \text { and } 6 \text { were examined for } \\
\text { conceptual fit with existing domain }\end{array}$ & $\begin{array}{l}\text { Two items had standardized } \\
\text { factor loadings }>0.4 \text { and }<0.6 \text { : } \\
\text { - FINANCE on the Psychological } \\
\text { domain }(0.47) \\
\text { - BURDEN on the Physical } \\
\text { domain }(0.49)\end{array}$ \\
\hline
\end{tabular}

Conclusions

BURDEN: evidence from the qualitative interviews had suggested that this is a separate aspect of QOL from the physical domain. In addition, the loading did not reach the criterion of $\geq 0.6$ for addition to a domain. BURDEN was therefore not added to the Physical domain

FINANCE: Conceptually different from psychological symptoms. May have loaded with them because the item captures stress due to financial situation. In addition, the loading did not reach the criterion of $\geq 0.6$ for addition to a domain. FINANCE was therefore not added to the Psychological domain

2 Determine if each new domain individually fit with MQOL-R CFA model of final version of MQOL-E
The 5 new domains (see Table 2) were added to the MQOL-R model one domain at a time (items were assigned to the new domains for which they were designed). For new domains that comprised a single item, the factor loading was fixed at 1.00 and the residual variance was fixed at zero.

Criteria

- $\mathrm{CFI}>0.95$

- RMSEA < 0.06

- Standardized factor loadings for all new items should be $\geq$ to all loadings for that item in Step 1

Domains retained in the separate analyses in Step 2 were added all at once to MQOL-R. Another model was fit without the Finance domain Criteria

- $\mathrm{CFI}>0.95$

- RMSEA $<0.06$

The model of MQOL-E from Step 3 was fit with the addition of a secondorder factor for overall QOL. This was repeated with the Finance domain removed.

The correlation of the overall factor with a single-item scale (MOOL-SIS) measure of global QOL was calculated.

Criteria

- $\mathrm{CFI}>0.95$

- RMSEA < 0.06
CFI range: $0.96-0.97$

- RMSEA range: 0.035-0.041

- Standardized factor loadings for all items for new domains were higher than in Step 1 (range: $0.72-0.88)^{\mathrm{a}}$
Model with Finance domain

- $\mathrm{CFI}=0.97$

- RMSEA $=0.033$

- Standardized factor loading range: $0.53-0.91^{\mathrm{b}}$

Model without Finance domain

- $\mathrm{CFI}=0.96$

- RMSEA $=0.033$

- Standardized factor loading range: $0.52-0.91^{\mathrm{b}}$

Model with Finance domain

- $\mathrm{CFI}=0.93$

- RMSEA $=0.042$

- Standardized factor loading of

Finance domain on secondorder factor: 0.25

Model without Finance domain

- $\mathrm{CFI}=0.93$

- RMSEA = 0.043

- Standardized factor loadings for both first and second order factors are shown in Fig. 1

- Correlations of the factors with the MQOL-SIS are reported in Table 4

Domain means, standard deviations and internal consistency estimates (Cronbach alphas) are reported in Table 5
All new domains fit with the existing MQOL-R, and were retained for step 3
The model with new domains had good fit, both with and without the Finance domain, providing support for the addition of the new domains
The second-order MQOL-E model with the Finance domain had overall acceptable model fit; although the CFI was slightly below 0.95 , the RMSEA clearly meets the criterion for good fit. The loading of the Finance domain on the second-order factor was very low.

The models with and without this domain had essentially the same fit (RMSEA difference of 0.001 and identical CFI). Since the Finance domain appeared to be a weak indicator of overall QOL and removing it did not result in poor model fit, the model without this domain was retained. This reduces the number of items and thus reduces participant burden.

${ }^{\mathrm{a}}$ The Burden, Environment, and Finance factors were each represented by a single item. The loadings of these items were therefore fixed at 1.00 ; comparisons with Step 1 are not relevant

${ }^{\mathrm{b}}$ This does not include the loadings for the single-item Burden, Environment and Finance factors since these loadings were fixed at 1.00 
strong theoretical support. Nevertheless, items with loadings between 0.4 and 0.6 were closely scrutinized for conceptual fit on existing MQOL-R domains. To assess whether it is justified to create a summary score of all of the domains, the usual criterion of a factor loading $\geq 0.4$ was used. Missing data were accommodated via full information maximum likelihood estimation. The total percentage of missing data was $18 \%$ when including all variables and $13 \%$ when including the variables in the final model.

Although McCaffrey et al (2016) included the concept of 'feeling like a burden' in the social domain (as did Cohen and Leis [8]), we were not certain whether it belonged in the social, psychological, or existential domain, or whether it would need to represent a separate domain. Assessment of this during creation of MQOL-R indicated it did not load sufficiently on any of the MQOL-R factors to justify its inclusion on any original domain, therefore it was included in the MQOL-E analyses as a separate factor. To assess the relevance of the financial domain, models both with and without the financial domain item were tested to determine the extent to which it made a unique contribution to the measurement of QOL.

MQOL-E subscale scores are calculated as the mean of the items measuring that domain. The MQOL-E summary score is the mean of the subscale scores (it does not include the global item). The Pearson correlations between the latent domains, second order factor, and a global measure of QOL were calculated for descriptive purposes. Cronbach's alpha ( $\alpha$ ) was calculated to assess internal consistency reliability; an alpha $\geq 0.7$ is considered acceptable [25].

\section{Results}

The results and conclusions of each step of the analyses are described in Table 3. None of the new items loaded $\geq 0.6$ on the MQOL- $R$ factors, suggesting that they could represent new domains. There was good model fit when each of the 5 new domains was added separately to MQOL-R, as well as when all new domains were added together $(\mathrm{CFI}=0.97$; $\mathrm{RMSEA}=0.033)$, supporting the correlated factor structure of MQOL-E. There was acceptable model fit for a second-order factor, supporting the creation of a MQOL-E summary score $(C F I=0.93$; RMSEA = 0.042). However, the financial domain was removed from the final MQOL-E because the standardized loading on the second-order factor was low $(0.254$, well below the 0.4 criterion) and its removal had little effect on the CFI and RMSEA of the first-order (CFI $=0.96$; RMSEA $=0.033)$ and second-order $(C F I=0.93$, RMSEA = $0.043)$ models. This suggests that the Finance domain is a weak indicator of overall QOL. In keeping with our goal of ensuring MQOL-E is as brief as possible, the model without this domain was retained.
The items and standardized factor loadings for the final model are shown in Fig. 1. For the second-order factor, factor loadings ranged from 0.40 for the Burden domain to .83 for the Existential domain.

Domain means, standard deviations, and Cronbach's alphas are shown in Table 4. Cronbach's alpha for the domains measured with more than one item for the final model ranged from 0.66 to 0.87 (Physical $=0.66$; Psychological $=0.85 ; \quad$ Existential $=0.78 ; \quad$ Social $=.87 ; \quad$ Healthcare $=0.76 ; \quad$ Cognitive $=0.79)$. Table 5 shows the correlations with the global item (MQOL-SIS) and interdomain correlations, all of which were statistically significant $(p<.001)$ except for the correlation between the Cognitive and Burden domains $(p>.05)$. The highest correlation is between the MQOL-SIS and the 2nd order factor $(r=.65)$. The correlations between the MQOLSIS and the domains are between .64 (Physical) and .30 (Social and Burden). The Healthcare domain has similar correlations with all of the other domains (.25-.34) except that it is low for Burden (.16). The Cognitive domain is most highly correlated with the Existential domain (.56). The Burden domain is most highly correlated with the Physical domain (.53). The Environment domain is most highly correlated with the Existential (.44) and Cognitive (.43) domains, with a particularly low correlation with the Burden domain (.07).

\section{Discussion}

This study demonstrates that the four-domain MQOL-R can be expanded to include four new domains that people at the end of life report as important contributors to their QOL [8]. MQOL-E more closely reflects the domains that palliative care patients report are important to their QOL than existing QOL questionnaires while remaining brief. While we believe that MQOL-R is sufficient and appropriate when a shorter measure is needed to reduce response burden, MQOL-E gives a more wellrounded or comprehensive description of the QOL of people with a life-limiting illness. While longer than MQOL-R (15 items) [19], at 21 items MQOL-E can be completed by many people who are seriously or terminally ill when a more comprehensive assessment of QOL is desired, such as when QOL is the primary outcome of interest in a study. Structuring the MQOL-E so that the first 15 items are MQOL-R allows for flexibility, such as comparison to studies using MQOL-R or, when collecting longitudinal data, to shorten the questionnaire for people who become unable to respond to the complete MQOL-E.

Factor analysis supports the creation of a summary score for MQOL-E based on the eight domains. We tested the construct validity of calculating a summary score because it is necessary for many purposes. However, we advise using a profile of the individual subscale 


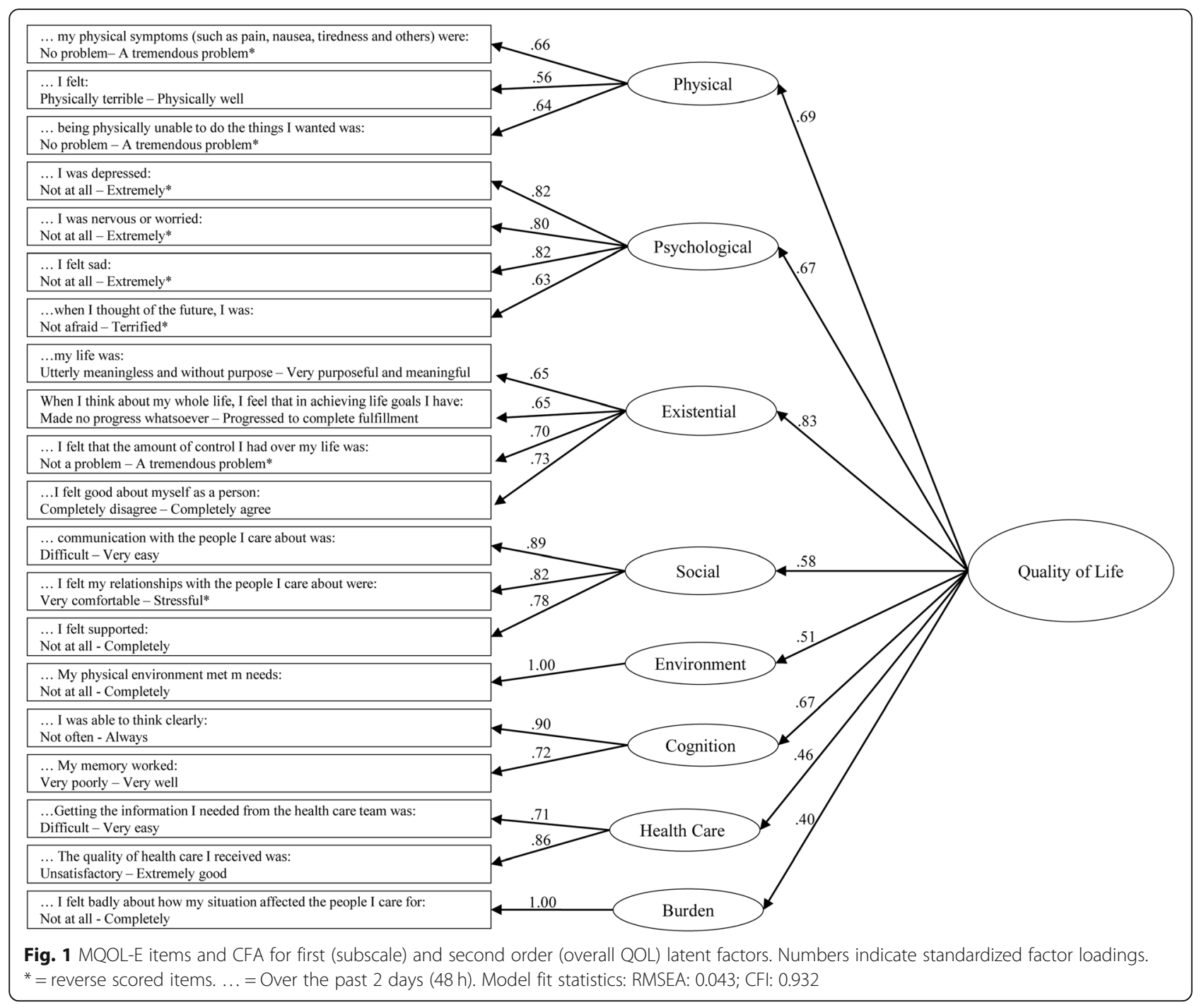

scores whenever possible, because they are more informative. For example, some domains may improve over time while others worsen, resulting in little change over time in the summary score despite important change occurring at the domain level. A strength of MQOL-E is that the factor analysis supports using separate scores for each of the eight domains. MQOL-E therefore provides information about a wider range of QOL contributors than instruments assessing only the physical, psychological, and social domains, as well as those that also include the spiritual or existential domain. The internal consistency of the Physical domain (Cronbach's alpha $=0.66$ ) was slightly below the accepted criterion of $\geq 0.7$. This may be because this domain is measured by items that reflect quite diverse aspects of physical health: 1) how much of a problem it is not to be able to do things you want to do; 2) how much of a problem physical symptoms are; and 3) a feeling of general physical wellbeing. To keep the instrument brief, each of these concepts is measured with a single item, likely lowering alpha.

The moderate correlation of the MQOL-E summary score with a single item measuring global QOL $(r=.65)$ suggests that MQOL-E measures QOL in a way that is related but not identical to a global assessment of QOL. The lower correlations between the global QOL item and the new domains added for MQOL-E underscores the value of measuring these aspects of QOL individually rather than assuming they will be reflected in a global assessment. The moderate correlations between several domains indicate that the experience in one life domain is related to the experience in other domains. The correlations of the new domains with other domains provide valuable new insights. For example, the Burden domain is most strongly correlated with the Physical domain $(r=.53)$, suggesting that physical wellbeing is more important than other domains in the extent to which one feels like a burden. However, since people with 
Table 4 Domain mean scores and standard deviations, Cronbach alpha

\begin{tabular}{lll}
\hline Domain & Mean (SD) & Cronbach Alpha \\
\hline Physical & $5.11(2.48)$ & 0.66 \\
Psychological & $6.83(2.49)$ & 0.85 \\
Existential & $7.19(1.99)$ & 0.78 \\
Social & $8.63(1.78)$ & 0.87 \\
Healthcare & $8.56(1.79)$ & 0.76 \\
Cognition & $8.23(1.97)$ & 0.79 \\
Burden & $4.58(3.59)$ & $\mathrm{N} / \mathrm{A}^{\mathrm{b}}$ \\
Environment & $8.27(2.16)$ & $\mathrm{N} / \mathrm{A}^{\mathrm{b}}$
\end{tabular}

${ }^{a}$ MQOL-E scores range from $0-10$, with 0 indicating the poorest QOL and 10 the best

${ }^{\mathrm{b}} \mathrm{Not}$ applicable because the domain is represented by a single item

significant cognitive impairment were not included in the study, it is possible that significant cognitive impairment is also important to feeling like a burden.

There are some limitations to the study. While it is a strength that MQOL-E was created using datasets collected in many cities in various care settings, because these studies used various versions of the questionnaire to test different items, the MQOL-E described in this paper was not tested in its exact final form. Another limitation is that some domains are measured with only one or two items. This may limit the scope of the content covered within each domain as well as its reliability. We imposed this limitation on MQOL-E to keep it brief and facilitate its completion throughout the illness trajectory and as close to the end of life as possible. Most (but not all) people who contributed data to the study had cancer, therefore it is unknown whether the domain structure will be the same when tested with samples with only non-cancer life-limiting illnesses. However, it is noteworthy that McCaffrey et al's systematic review found that aspects of QOL do not differ according to diagnosis, care setting, or living arrangement [3]. A strength of MQOL-E is that it was developed simultaneously in English and French. However, the potential for differences in the structure of the English and French versions could not be investigated with the present data (i.e. testing for measurement invariance) and should be investigated in future studies. Furthermore, people in the recruitment settings who spoke neither language are not represented in this study. In order to allow QOL instruments to be completed even by people who are quite weak, or have difficulty seeing or are illiterate, it is important that they can be read aloud to the respondent, as well as self-completed by those without these limitations. In this study we combined data from both methods of administration to use data from the widest variety of respondents. Because we did not have enough data to test whether MQOL-E's structure is the same for different modes of administration, future studies should examine this.

MQOL-E includes seven of the eight domains found by McCaffrey et al to have solid evidence for being important to the QOL of palliative care patients [3]. The domain not covered is preparation for death and afterward. After initially considering including this domain, we later decided to exclude it because preparation is an activity, whereas MQOL-E is intended to measure QOL as a subjective experience. If preparation enhances QOL, we expect that this would be through improving other domains, such as (feeling like a) burden, social, or existential.

As illnesses progress, healthcare takes on an increasingly large role in a person's life. Measures of the occurrence (or not) of specific aspects of healthcare are usually considered person-centred experience or process measures, while assessments of QOL are considered to be outcome measures. Nevertheless, MQOL-E includes healthcare as a domain of QOL for two reasons. First, healthcare was found to be important to QOL in the

Table 5 Pearson correlations between the second order factor, latent domains, and the single-item global measure of quality of life (MQOL-SIS)

\begin{tabular}{|c|c|c|c|c|c|c|c|c|}
\hline & MQOL-SIS & Physical & Psycho-logical & Existen-tial & Social & Health-care & Cognitive & Burden \\
\hline 2nd order factor & .65 & & & & & & & \\
\hline \multicolumn{9}{|c|}{ 1st order factor model } \\
\hline Physical & .64 & & & & & & & \\
\hline Psychological & .43 & .62 & & & & & & \\
\hline Existential & .52 & .46 & .57 & & & & & \\
\hline Social & .30 & .28 & .35 & .52 & & & & \\
\hline Healthcare & .32 & .27 & .25 & .34 & .28 & & & \\
\hline Cognitive & .31 & .36 & .41 & .56 & .45 & .34 & & \\
\hline Burden & .30 & .53 & .33 & .34 & .20 & .16 & .20 & \\
\hline Environment & .32 & .27 & .26 & .44 & .32 & .33 & .43 & .07 \\
\hline
\end{tabular}


Cohen and Leis study [8] on which MQOL-E is based and in 13/24 studies in the McCaffrey et al review [3]. Second, the MQOL-E healthcare items involve a subjective rating of quality, rather than simply the occurrence of aspects of healthcare as found in patient experience instruments.

We created a new domain, Burden. Not only is feeling like a burden important to QOL, it is one of the major reasons that people request a hastened death [26-28], and therefore important in QOL assessment. We initially expected this item to load on the social domain, but it loaded moderately on the physical domain. We believe this is so because the worse their physical condition, the more help the respondent is likely to need from others, potentially leading them to feel more like a burden. McCaffrey et al [3] note this relationship in describing the personal autonomy domain, but also mention the concept of burden in describing the preparatory (taking care of one's affairs is perceived to relieve burden on family) and social domains. While relevant to several domains, this item was kept as a separate one in MQOL-E to ease interpretation, and to maintain the items measuring the physical domain identical to those in MQOL-R.

It is not clear in the literature whether finances are an important contributor to the QOL of people with advanced disease. We considered one item about finances, but ultimately excluded it because it loaded moderately on the psychological domain (possibly because it assessed stressfulness of finances), making it difficult to interpret. If finances are important because they add to feeling like a burden, as has been found in other studies [29], that is captured by our Burden domain. We can only speculate as to why financial concerns were not identified as a distinct domain in measuring overall QOL in our study. It may be that our studies were conducted in Canada, where most healthcare is paid for by the government. Alternatively, as another study in Canada found finances to be a contributor to family caregiver QOL, it may be that many people with very advanced disease leave that concern to their families [30]. A version of MQOL-E including the financial item is available for use when measuring finances as a source of stress is considered important, but this item should not be used in computing the summary score.

The correlations of the new domains with the MQOLSIS, a global item measuring overall QOL, were .30-.32. These correlations are quite a bit lower than those for the Physical (.64), Psychological (.42), and Existential (.52) domains. However, these correlations are similar to that for the Social domain (.30), which is generally accepted as important to include in QOL instruments [2]. Since the Social domain has a similar correlation but is reported as important by almost all palliative care patients reporting on what is important to their QOL [3], the lower correlations for the new domains may reflect the limitations of measuring QOL with a single global item.

Comparing the domains coverage of MQOL-E to two other widely used QOL measurement systems, the FACIT-PAL (46 items) and its shorter version, FACITPAL-14 (14 items) [31, 32], and the EORTC QLQ-C30 and its version for palliative care, the EORTC QLQC15-PAL [10, 33], MQOL-E covers more of the domains found in the McCaffrey et al review [3]. The FACITPAL comes closest to the domain coverage of MQOL-E, including cognition and feeling like a burden, but it does not cover healthcare or environment. At 46 items it is more than twice as long as MQOL-E. The FACIT-PAL14 touches upon similar domains to its parent, but unlike the longer version does not include cognition. The EORTC QLQ-C15-PAL touches only the physical, psychological, and personal autonomy domains, while the longer instrument additionally touches on the social and cognition domains but does not cover the spiritual/existential or healthcare domains, nor do they include the concept of burden. None of the instruments covers the preparatory domain, although we do not see that as a drawback, for the reasons explained above. For consideration of some other differences, see Cohen et al. [19].

We are further exploring the psychometric properties of MQOL-E in an ongoing study with a new population, including a confirmatory factor analysis of MQOL-E in its final version, and assessing test-retest reliability and responsiveness to change. Future validation of MQOL-E should include investigation of convergent and discriminant validity and of measurement invariance of the English and French versions as well as mode of administration.

\section{Conclusion}

Given the various life domains that people living with life-threatening illness find important to their QOL, the creation of MQOL-E, by adding the four domains of healthcare, cognitive functioning, (feeling like a) burden, and environment to the four domains covered by MQOL-R (physical, psychological, existential, social), is an important advance in QOL measurement. The statistical confirmation of eight distinct domains is critical since this permits measurement of different aspects of QOL. This study also provides evidence of the validity of a summary score, when desired. MQOL-E is a more comprehensive measure of QOL compared to existing instruments. A strength of the instrument is that it includes MQOL-R, which allows comparison to studies using MQOL-R. We expect that use of MQOL-E will advance our understanding of the well-studied and new facets of the QOL of people with a variety of lifethreatening illnesses. 


\section{Supplementary information}

Supplementary information accompanies this paper at https://doi.org/10. 1186/s12904-019-0473-y

Additional file 1. Research ethics committees.

\section{Abbreviations}

CFI: Comparative fit index; CHF: Congestive heart failure; COPD: Chronic obstructive pulmonary disease; EORTC QLQ-C15-PAL: European Organization for Research and Treatment of Cancer - Quality of Life Questionnaire Core15-Palliative Care; EORTC QLQ-C30: European Organization for Research and Treatment of Cancer - Quality of Life Questionnaire - Core30; FACITPAL: Functional Assessment of Chronic Illness Therapy - Palliative Care Scale; MQOL SIS: McGill Quality of Life Questionnaire Single Item Scale; MQOL: McGill Quality of Life Questionnaire; MQOL-E: McGill Quality of Life Questionnaire- Expanded; MQOL-R: McGill Quality of Life Questionnaire Revised; QOL: Quality of life; RMSEA: Root mean square error of approximation

\section{Acknowledgements}

This paper is dedicated to Terry Bunston and Jane Poulson, both deceased. They played essential roles in the initial efforts to create MQOL-Expanded. We thank those who ran study $G$ at various sites: Cécile Charbonneau, Gillian Fyles, Ray Viola, Jane Poulson, Lucie Laplante, Val Zemlak, Justine Farley. We thank Daren Heyland, Andrew Day, and Xuran Jiang for providing dataset F.

\section{Authors' contributions}

All authors contributed to the writing of the manuscript. All authors read and approved the final version of the manuscript. SRC conceived of the need for MQOL-E, designed the study to develop and determine the acceptability of the pool of potential MQOL-E items, designed and ran study G, supervised study $E$, and developed the analysis plan for this manuscript. RS and LBR developed and carried out the analysis plan. AL designed the study to develop and determine the acceptability of the pool of potential MQOL-E items and designed and ran study G. JS designed and collected data for and provided dataset $\mathrm{E}$, designed to evaluate an interim version of MQOL-E. PP and DRK were involved in developing the new items and data collection for dataset G. AMG provided input in developing the analysis plan.

\section{Funding}

The initial project to expand MQOL was funded by the Canadian Cancer Society Research Institute (CCSRI) (\#8057) with funds donated to the Canadian Cancer Society, as was the study for dataset G (\#012201). Dataset E was collected with no specific grant from any funding agency in the public, commercial, or not-for-profit sectors. Funding for dataset $F$ is acknowledged in the primary publication from that grant [19]. None of the funders of the research had a role in this study or the studies used in this report, other than providing unrestricted funds. This research was undertaken, in part, thanks to funding from the Canada Research Chairs program. Dr. Sawatzky holds a Canada Research Chair in Person-Centred Outcomes.

\section{Availability of data and materials}

The datasets used and/or analysed during the current study are available from the corresponding author on reasonable request.

\section{Ethics approval and consent to participate}

The original studies which generated these datasets were approved by university and/or hospital human research ethics committees at all of the data collection sites. These are listed in the Additional file 1 available online. Written informed consent was obtained from all participants.

\section{Consent for publication}

Not applicable

\section{Competing interests}

S. Robin Cohen is an Associate Editor of this journal. The Authors declare that they have no other competing interests.

\section{Author details}

'Departments of Oncology and Medicine, McGill University, Montréal, Canada. ${ }^{2}$ Lady Davis Research Institute of the Jewish General Hospital, Palliative Care Research, room E8.06, 3755 Côte Ste. Catherine Road, Montréal, Québec H3T 1E2, Canada. ${ }^{3}$ Centre for Health Evaluation and Outcomes Sciences, Providence Health Care Research Institute, Vancouver, British Columbia, Canada. ${ }^{4}$ Department of Community Health \& Epidemiology, College of Medicine, University of Saskatchewan, Saskatoon, Saskatchewan, Canada. ${ }^{5}$ Daiichi Sankyo Inc, Basking Ridge, NJ, USA. ${ }^{6}$ Retired RN, MSN, Vancouver, Canada. ${ }^{7}$ Departments of Family Practice and Urologic Sciences, University of British Columbia, Vancouver, British Columbia, Canada. ${ }^{8}$ Human Early Learning Partnership, School of Population and Public Health, University of British Columbia, Vancouver, Canada. ${ }^{9}$ School of Nursing, Trinity Western University, Langley, British Columbia, Canada.

Received: 13 July 2018 Accepted: 26 September 2019

Published online: 31 October 2019

\section{References}

1. Basic Documents. Constitution. 48th ed. Italy: World Health Organization; 2014. p. 1.

2. Albers G, Echteld MA, de Vet HC, Onwuteaka-Philipsen BD, van der Linden $\mathrm{MH}$, Deliens L. Content and spiritual items of quality-of-life instruments appropriate for use in palliative care: a review. J Pain Symptom Manag. 2010;40(2):290-300.

3. McCaffrey N, Bradley S, Ratcliffe J, Currow DC. What Aspects of Quality of Life Are Important From Palliative Care Patients' Perspectives? A Systematic Review of Qualitative Research. Journal of Pain and Symptom Management. 2016:52(2):318-328.e5.

4. Cohen SR, Mount BM, Tomas JJ, Mount LF. Existential well-being is an important determinant of quality of life. Evidence from the McGill quality of life questionnaire. Cancer. 1996;77(3):576-86.

5. Cohen SR, Mount BM, Bruera E, Provost M, Rowe J, Tong K. Validity of the McGill quality of life questionnaire in the palliative care setting: a multiCentre Canadian study demonstrating the importance of the existential domain. Palliat Med. 1997;11(1):3-20.

6. Albers $G$, Echteld MA, de Vet $\mathrm{HC}$, Onwuteaka-Philipsen $B D$, van der Linden $\mathrm{MH}$, Deliens L. Evaluation of quality-of-life measures for use in palliative care: a systematic review. Palliat Med. 2010;24(1):17-37.

7. Stiel S, Pastrana T, Balzer C, Elsner F, Ostgathe C, Radbruch L. Outcome assessment instruments in palliative and hospice care-a review of the literature. Support Care Cancer. 2012;20(11):2879-93.

8. Cohen SR, Leis A. What determines the quality of life of terminally ill cancer patients from their own perspective? J Palliat Care. 2002;18(1):48-58.

9. Shahidi J, Bernier N, Cohen SR. Quality of life in terminally ill cancer patients: contributors and content validity of instruments. J Palliat Care. 2010;26(2):88-93.

10. Groenvold M, Petersen MA, Aaronson NK, Arraras JI, Blazeby JM, Bottomley A, et al. The development of the EORTC QLQ-C15-PAL: a shortened questionnaire for cancer patients in palliative care. Eur J Cancer. 2006;42(1):55-64.

11. Winstanley JB, White EG, Boyle FM, Thompson JF. What are the pertinent quality-of-life issues for melanoma cancer patients? Aiming for the development of a new module to accompany the EORTC core questionnaire. Melanoma Res. 2013;23(2):167-74.

12. Lee GL, Pang GSY, Akhileswaran R, Ow MYL, Fan GKT, Wong CCF, et al. Understanding domains of health-related quality of life concerns of Singapore Chinese patients with advanced cancer: a qualitative analysis. Support Care Cancer. 2016;24(3):1107-18.

13. Shahidi J. Construct validity of the quality of life in life-threatening illnesspatient questionnaire (QOLLTI-P) in cancer patients: McGill University; 2010.

14. Mystakidou K, Tsilika E, Kouloulias V, Parpa E, Katsouda E, Kouvaris J, et al. The "palliative care quality of life instrument (PQLI)" in terminal cancer patients. Health Qual Life Outcomes. 2004;2:8.

15. Steinhauser KE, Bosworth HB, Clipp EC, McNeilly M, Christakis NA, Parker J, et al. Initial assessment of a new instrument to measure quality of life at the end of life. J Palliat Med. 2002;5(6):829-41.

16. Wettergren $L$, Lindblad AK, Glimelius B, Ring L. Comparing two versions of the schedule for evaluation of individual quality of life in patients with advanced cancer. Acta Oncol. 2011;50:648-52.

17. Padilla GV, Ferrell B, Grant MM, Rhiner M. Defining the content domain of quality of life for cancer patients with pain. Cancer Nurs. 1990;13(2):108-15. 
18. Waldron D, O'Boyle CA, Kearney M, Moriarty M, Carney D. Quality-of-life measurement in advanced cancer: assessing the individual. J Clin Oncol. 1999;17(11):3603-11.

19. Cohen SR, Sawatzky R, Russell LB, Shahidi J, Heyland DK, Gadermann AM. Measuring the quality of life of people at the end of life: the McGill quality of life questionnaire-revised. Palliat Med. 2017;31(2):120-9.

20. Heyland DK, Cook DJ, Rocker GM, Dodek PM, Kutsogiannis DJ, Skrobik Y, et al. The development and validation of a novel questionnaire to measure patient and family satisfaction with end-of-life care: the Canadian health care evaluation project (CANHELP) questionnaire. Palliat Med. 2010;24(7):682-95.

21. Muthén B, Muthén L. MPlus (version 8). Los Angeles: Statmodel; 2017.

22. Hu L, Bentler PM. Cutoff criteria for fit indexes in covariance structure analysis: conventional criteria versus new alternatives. Struct Equ Model. 1999;6:1-55.

23. Brown TA. Confirmatory factor analysis for applied research. New York: Guilford Press; 2015.

24. Stevens JP. Applied multivariate statistics for the social sciences (2nd edition). Hillsdale: Erlbaum; 1992.

25. Reeve BB, Wyrwich KW, Wu AW, Velikova G, Terwee CB, Snyder CF, et al. ISOQOL recommends minimum standards for patient-reported outcome measures used in patient-centered outcomes and comparative effectiveness research. Qual Life Res. 2013;22:1889-905.

26. Hudson PL, Kristjanson LJ, Ashby M, Kelly B, Schofield P, Hudson R, et al. Desire for hastened death in patients with advanced disease and the evidence base of clinical guidelines: a systematic review. Palliat Med. 2006; 20(7):693-701.

27. Monforte-Royo C, Villavicencio-Chávez C, Tomás-Sábado J, Mahtani-Chugani V, Balaguer A. What lies behind the wish to hasten death? A systematic review and meta-ethnography from the perspective of patients. PLoS One. 2012;7(5):e37117.

28. Rodríguez-Prat A, Balaguer A, Booth A, Monforte-Royo C. Understanding patients' experiences of the wish to hasten death: an updated and expanded systematic review and meta-ethnography. BMJ Open. 2017;7(9): e016659.

29. Kim SH, Choi YS, Lee J, Oh SC, Yeom CH, Lee MA, et al. Reliability and validity of the hospice quality of life scale for Korean cancer patients. J Pain Symptom Manag. 2009;37(2):156-67.

30. Cohen SR. Defining and measuring quality of life in palliative care. In: Bruera E, Portenoy RK, editors. Topics in Palliative Care. 5. New York: Oxford University Press; 2001. p. 137-56.

31. Lyons KD, Bakitas M, Hegel MT, Hanscom B, Hull J, Ahles TA. Reliability and validity of the functional assessment of chronic illness therapy-palliative care (FACIT-pal) scale. J Pain Symptom Manag. 2009:37(1):23-32.

32. Zeng L, Bedard G, Cella D, Thavarajah N, Chen E, Zhang LY, et al. Preliminary results of the generation of a shortened quality-of-life assessment for patients with advanced Cancer: the FACIT-pal-14. J Palliat Med. 2013;16(5):509-15.

33. Aaronson NK, Ahmedzai S, Bergman B, Bullinger M, Cull A, Duez NJ. The European Organization for Research and Treatment of Cancer QLQ-C30: a quality-of-life instrument for use in international clinical trials in oncology. J Natl Cancer Inst. 1993:85(5):365-76.

\section{Publisher's Note}

Springer Nature remains neutral with regard to jurisdictional claims in published maps and institutional affiliations.

Ready to submit your research? Choose BMC and benefit from:

- fast, convenient online submission

- thorough peer review by experienced researchers in your field

- rapid publication on acceptance

- support for research data, including large and complex data types

- gold Open Access which fosters wider collaboration and increased citations

- maximum visibility for your research: over $100 \mathrm{M}$ website views per year

At BMC, research is always in progress.

Learn more biomedcentral.com/submissions 